\title{
Vulnerable Road Users in Mexico: The Perils of Pedestrian Mobility in Developing Countries
}

\author{
Arturo Cervantes*, Trejo Liliana, Camarillo Rodríguez and Isaac Castañeda Alcántara \\ Professor, Faculty of Health Sciences, Universidad Anahuac, Mexico
}

Submission: February 17, 2018; Published: March 26, 2018

*Corresponding author: Arturo Cervantes, Professor, Faculty of Health Sciences, Universidad Anahuac, Mexico; Email: arturocer@gmail.com

Abbreviations: LMIC: Low-and-Middle-Income Countries; RTI's: Road Traffic Injuries; VRU's: Vulnerable Road Users; ICD: International

Classification of Diseases

\section{Background}

Road deaths are projected to increase globally, from 1.2 million in 2002 to 2.1 million in 2030, primarily due to increased motor vehicle fatalities associated with economic growth in low-and-middle-income countries (LMIC) [1]. Road traffic injuries (RTI's) are a major global public health crisis, and are projected to become the fifth leading cause of death in the world by the year 2030 [2-4]. WHO reminds us that almost one half of the 1.2 million fatalities occurring each year in roads concern Vulnerable Road Users (pedestrians, cyclists and motorcyclists), with children and the elderly being overrepresented among victims [5,6]. In our previous analyses of temporal and geographic trends of mortality from road traffic injuries (RTI's) in Mexico, our group identified that, in spite of a steady decrease over the 1998 - 2013 period, mortality rates remained high in all Vulnerable Road Users (VRU's) over the age of 50 years [7]. This previous work evidenced the limitations regarding the grouping of all vulnerable road users into one category, which could obscure differential risk patterns among pedestrians, cyclists, and motorcyclists. Within the context of the United Nations Decade of Action for Road Safety, the main objective is to examine the demographic, temporal and state level patterns of pedestrian road traffic fatalities that occurred from 2000 to 2015 in Mexico, and to provide estimates of fatality rates among sex-age groups, in order to identify those at highest risk $[8,9]$.

\section{Methods}

This is a population-based retrospective study. We used two sources of public data: official mortality records from 20002015, which provide detailed data on all deaths, classified according to the International Classification of Diseases, and national population projections 2000-2015 at national and state levels [10-12]. We estimated RTI rates and specific pedestrian fatality rate trends for different sex-age groups at national and state levels, by using a logistic regression model [13]. We also compared pedestrian fatality rate trends and overall annual incidence rates at state level, for pedestrians 60 years and older. The models were fitted with the software R version 3.2.3 [14]. To find the regression parameters we used maximum likelihood estimation and for every fit we performed the chisquare goodness of fit test, and checked the significance of each parameter with the $t$ statistic, controlling the level of significance at $\alpha=0.05$ [15].

\section{Data Sources}

Two sources of public data were used in the current project:

a) official mortality records of Mexico 2000-2015, which provide detailed data on all deaths, including the cause (classified according to the International Classification of Diseases (ICD-10)(WHO, 2011), exact time of death, demographic information of the deceased, and

b) National population projections 2000-2015 at national and state level. From the mortality database, we selected the records of pedestrian's casualties, [V02-V04 (.1, .9), V09.2, V09.3, V09.9, (Table 1)](INEGI \& CEMECE, 1998) and we obtained the mortality's counts differentiating by state of occurrence, sex and age groups, which were grouped into 10 categories $(0-9,10-14,15-19,20-24,25-29,30-39,40-$ $49,50-59,60-74$ and 75 or older). Likewise, we calculated the population's counts differentiating by state, sex and age groups. By combining both sources of information, we obtained a data set with the number of inhabitants as well as the number of fatalities at national, sub national level, by sex-age group, for each year included in the study. 
Table 1: ICD-10 codes associated with pedestrian fatalities.

\begin{tabular}{|c|c|}
\hline Type of Accident & ICD-10 Codes \\
\hline $\begin{array}{c}\text { Pedestrian injured in collision } \\
\text { with two or three - wheeled } \\
\text { motor vehicle }\end{array}$ & V02 $(.1, .9)$ \\
\hline $\begin{array}{c}\text { Pedestrian injured in collision } \\
\text { with car, pick-up truck or van }\end{array}$ & V03 $(.1, .9)$ \\
\hline $\begin{array}{c}\text { Pedestrian injured in collision } \\
\text { with heavy transport vehicle or } \\
\text { bus }\end{array}$ & V04 $(.1, .9)$ \\
\hline $\begin{array}{c}\text { Pedestrian injured in other and } \\
\text { unspecified transport accidents }\end{array}$ & V09.2, V09.3, V09.9 \\
\hline
\end{tabular}

\section{Statistical Analysis}

We analyzed the pedestrian fatalities trends for each sexage group and state by using a logistic regression model. The number of fatalities in each groups under study i during year $t$, denoted Yit, and is assumed to follow a binomial distribution see Equation (1):

$$
Y_{t} \sim \operatorname{Bin}\left(n i_{t}, \pi i_{t}\right)
$$

Where nt, is the population count of the given sex-age group $\mathrm{i}$ during year $\mathrm{t}$ and $\pi \mathrm{it}$ is the probability that an individual from this subpopulation dies during a traffic accident being parameterized by the following function Equation (2):

$$
\log (\pi i t / 1-\pi i t)=\beta 0 i+\beta j i
$$

The parameter $\beta 0 \mathrm{i}$ can be interpreted as the log-odds of being killed in the group $i$ parameter during year 2000 and the parameter $\beta \mathrm{ji}$ indicate how these log-odds change (per year) [7]. The models were fitted with the software $\mathrm{R}$ version 3.2.3 [14]. To find the regression parameters we used maximum likelihood estimation [13]. For every fit we performed the chi- square goodness of fit test, and checked the significance of each parameter with the $t$ statistic, controlling the level of significance at $\alpha=0.05$ [15].

\section{Overall Annual Incidence Rates}

We calculated the overall annual incidence rate per each state by dividing the mortality count over all the period of study between the numbers of persons at risk during the same period.

\section{Results}

From 2000 to 2015, Mexico registered 253,582 road deaths, of which 81,061 were pedestrians (32\%). During the period, population size and motor vehicles had a sustained annual growth of $1.2 \%$ and $6.4 \%$ respectively. The odds ratios for pedestrian deaths are greater for males in comparison with females $(\mathrm{OR}=$ 3.6, C.I 3.58 - 3.70). RTI mortality rates in all age-sex groups decreased significantly during the period. Irrespective of gender groups, as age increases, pedestrian mortality rates increase, with the highest fatality rates observed in adults in age groups 60-75 and 75 and older. Analyses at sub-national (state) level show a large heterogeneity of pedestrian fatality rates for adults age 60 and older. Among males older than 60 years, in half of the Mexican states $(\mathrm{N}=16)$ there is a significant decreasing trend in fatality rates. In 12 states, pedestrian fatality rates are stable over the period, and in four states, there is a significant increase in fatality rates over the period. Between 2000-2015, were registered in Mexico a total of 253,582 casualties, of which, pedestrian fatalities accounted for almost a third $(n=81,061$, $32 \%$; (Table 2), fatalities in protected users (which includes users of motorized vehicles) accounted for $62.7 \% \mathrm{n}=159,094$; (Table 1) and motorcyclist and cyclist's fatalities accounted for 5.1\%; n=1,299 (Table 2). During the same period, the population and vehicle numbers showed a sustained increase, observing an annual rate growth of $1.22 \%$ and $6.5 \%$ respectively (Table 2 ).

\begin{tabular}{|c|c|c|c|c|c|c|c|c|}
\hline \multirow[b]{3}{*}{ Year } & \multirow[b]{3}{*}{ Population } & \multirow[b]{3}{*}{ Vehicles } & \multicolumn{6}{|c|}{ Pedestrian Fatalities } \\
\hline & & & \multicolumn{2}{|c|}{ Males } & \multicolumn{2}{|c|}{ Females } & \multicolumn{2}{|c|}{ Total } \\
\hline & & & Count & Rate & Count & Rate & Count & Rate \\
\hline 2000 & $100,895,811$ & $15,611,916$ & 4,291 & 8.64 & 1,216 & 2.37 & 5,509 & 5.46 \\
\hline 2001 & $102,122,295$ & $17,300,530$ & 4,181 & 8.32 & 1,295 & 2.50 & 5,480 & 5.37 \\
\hline 2002 & $103,417,944$ & $18,784,594$ & 4,112 & 8.09 & 1,206 & 2.29 & 5,328 & 5.15 \\
\hline 2003 & $104,719,891$ & $19,806,960$ & 4,077 & 7.94 & 1,263 & 2.37 & 5,348 & 5.11 \\
\hline 2004 & $105,951,569$ & $20,878,438$ & 3,880 & 7.47 & 1,192 & 2.21 & 5,076 & 4.79 \\
\hline 2005 & $107,151,011$ & $22,138,478$ & 3,998 & 7.62 & 1,157 & 2.12 & 5,159 & 4.81 \\
\hline 2006 & $108,408,827$ & $24,907,229$ & 4,096 & 7.73 & 1,123 & 2.03 & 5,220 & 4.82 \\
\hline 2007 & $109,787,388$ & $26,747,197$ & 3,775 & 7.04 & 1,041 & 1.85 & 4,819 & 4.39 \\
\hline 2008 & $111,299,015$ & $29,287,903$ & 3,815 & 7.02 & 1,117 & 1.96 & 4,934 & 4.43 \\
\hline 2009 & $112,852,594$ & $30,890,136$ & 4,010 & 7.27 & 1,135 & 1.97 & 5,147 & 4.56 \\
\hline 2010 & $114,255,557$ & $31,636,258$ & 3,728 & 6.68 & 1,055 & 1.80 & 4,786 & 4.19 \\
\hline 2011 & $115,682,867$ & $33,278,309$ & 3,773 & 6.68 & 1,080 & 1.83 & 4,868 & 4.21 \\
\hline
\end{tabular}

Table 2: Population and vehicle nationwide by year and pedestrian fatalities numbers and rates per 100,000 populations, Mexico $2000-20151$. 
Juniper Online Journal of Public Health

\begin{tabular}{|l|l|c|c|c|c|c|c|c|}
\hline 2012 & $117,053,751$ & $34,874,655$ & 4,023 & 7.04 & 1,142 & 1.91 & 5,177 & 4.42 \\
\hline 2013 & $118,395,053$ & $36,743,331$ & 3,798 & 6.57 & 1,012 & 1.67 & 4,816 & 4.07 \\
\hline 2014 & $119,713,203$ & $38,025,389$ & 3,794 & 6.49 & 1,017 & 1.66 & 4,821 & 4.03 \\
\hline 2015 & $121,005,816$ & $40,205,671$ & 3,587 & 6.49 & 978 & 1.66 & 4,573 & 3.78 \\
\hline Total & & & 62,938 & & 18,029 & & 81,061 & \\
\hline
\end{tabular}

${ }^{1}$ In 94 pedestrian fatalities the information about the gender of the victim was missing.

\section{Analysis of Pedestrian Collisions}

By using the ICD-10 codes (Table 1) we examined the collision mechanisms in pedestrians. In most of the deaths, the information was missing $(70,917 / 81,061,87.9 \%)$, in $9.4 \%$ $(n=7,650)$ the pedestrian suffered a collision with a car, pickup truck or van, in $2.4 \%$ of the cases suffered a collision with heavy transport vehicle or bus, and $0.6 \%(n=512)$ were caused by a collision with two-or three-wheeled motor vehicle. Between 2000 and 2015, fatality pedestrian rates declined from $39.3 \%$ to $28.5 \%$ which was significative (Table 2), evidenced by the negative slope obtained by fitting the data with a logistic regression model $(\beta 1=-0.022$, CI $95 \%=[-0.023,-0.020] \mathrm{p}<$ 0.001).

\section{Analysis of Pedestrian Fatalities by Gender and Age Groups}

When comparing by sex group, the odds of fatal accidents are greater for males in comparison with females $(\mathrm{OR}=3.6$, CI $95 \%=$ $[3.58,3.7])$. Out of 81,061 pedestrian fatalities, males accounted for $77.6 \%$ and females accounted for $22.2 \%$ (Table 2). Between males, there is an increase in fatality rates as the age increases (Figure 1). In comparison, the fatality rates in females remained relatively constant in the youngest and start to increase as an age function since the 25-29 age group and older (Figure 1). Table 3 presents the parameter estimates obtained for the logistic regression model for each sex-age group under study. The intercept (which must be interpreted in the log it scale) is directly related with the mortality rate in year 2000 and it must be transformed to rate by means of the equation presented in Methods.

The value of the slope indicates the strength of a decrease (negative) or increase (positive) in the mortality rate during 2000-2015. In all age-sex groups, the sign of the slope suggests, that there was a significant decrease in fatality rates during the period of the study $(\mathrm{p}<0.01)$, except for the group of females between 25-29 years of age. In this group, fatality rates remained constant during the period of study $(\beta 1=-0.012, \mathrm{CI} 95 \%=[-0.03$, $0.002])$. In both sex-groups, the highest reduction in fatality rates was observed in the 10-14 years old group. Despite that almost all age-sex groups had a significant reduction of their fatality rates over the period of study; in 2015 the fatality rates between the male adults of 60-74 years of age and 75 and over are still high: 13.9 and 24.2 deaths per 100,000 populations respectively. In view of these results, we will examine further the regional trends in the adults aged 60 and over, which in Mexico are denoted as older adults.

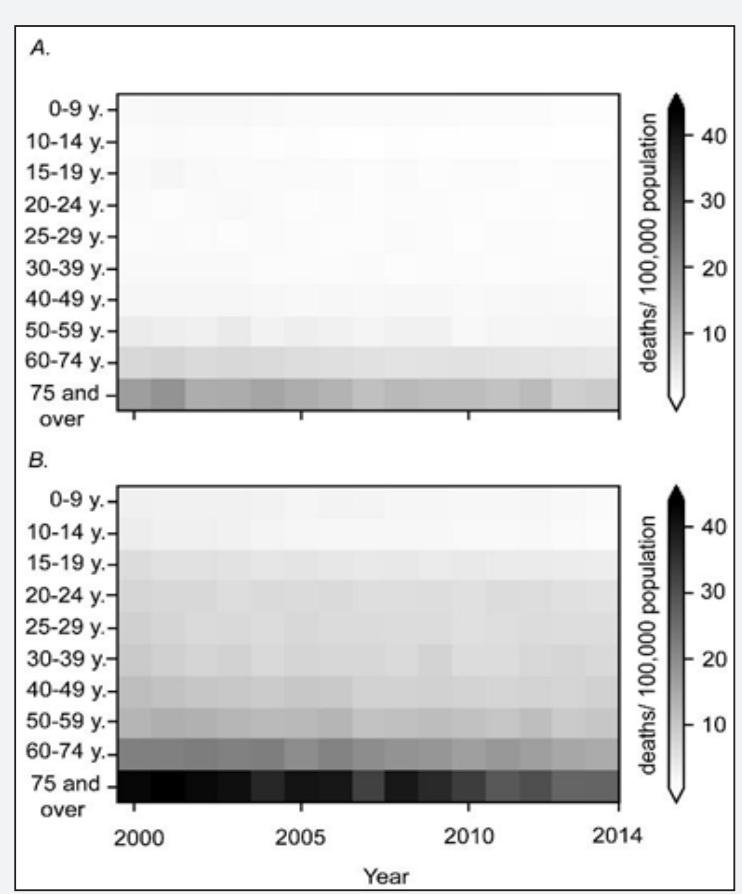

Figure 1: Pedestrian fatality rates in females per age groups (100,000 population). B. Pedestrian fatality rates in males per age groups (100,000 population). 
Table 3: Estimates of the parameters obtained by fitting the data across the different sex-age groups with a logistic regression model.

\begin{tabular}{|c|c|c|c|c|c|c|c|}
\hline \multirow{2}{*}{$\begin{array}{l}\text { Age-Sex } \\
\text { Groups }\end{array}$} & \multirow{2}{*}{ Parameter } & \multirow{2}{*}{ B } & \multirow{2}{*}{ Rate 100,000 pop. } & \multirow{2}{*}{ Standard error } & \multirow{2}{*}{ Sig. } & \multicolumn{2}{|c|}{ Confidence Interval } \\
\hline & & & & & & Inf & Sup \\
\hline & \multicolumn{7}{|c|}{ Males } \\
\hline \multirow[t]{2}{*}{$0-9$} & Intercept 2000 & -10.34 & 3.04 & 0.03 & 0 & -10.41 & -10.93 \\
\hline & Slope 2000-2015 & & & 0 & & & -0.04 \\
\hline \multirow[t]{2}{*}{ Oct-14 } & Intercept 2000 & -10.22 & 3.59 & 0.04 & 0 & -10.3 & -10.13 \\
\hline & Slope 2000-2015 & -0.07 & & 0.01 & & -0.08 & -0.06 \\
\hline \multirow[t]{2}{*}{$15-19$} & Intercept 2000 & -9.68 & 6.5 & 0.03 & 0 & -9.79 & -9.61 \\
\hline & Slope 2000-2015 & -0.04 & & 0 & & & -0.03 \\
\hline \multirow[t]{2}{*}{$20-24$} & Intercept 2000 & -9.51 & 7.46 & 0.03 & 0 & -9.58 & -9.45 \\
\hline & Slope 2000-2015 & -0.02 & & 0 & & & -0.01 \\
\hline \multirow[t]{2}{*}{$25-29$} & Intercept 2000 & -9.46 & 8.5 & 0.03 & 0 & -9.54 & -9.4 \\
\hline & Slope 2000-2015 & -0.02 & & 0 & & & -0.01 \\
\hline \multirow[t]{2}{*}{$30-39$} & Intercept 2000 & -9.37 & 9.59 & 0.02 & 0 & -9.43 & -9.33 \\
\hline & Slope 2000-2015 & -0.02 & & 0 & & & -0.01 \\
\hline \multirow[t]{2}{*}{$40-49$} & Intercept 2000 & -9.1 & 11.65 & 0.02 & 0 & -9.17 & -9.05 \\
\hline & Slope 2000-2015 & -0.03 & & 0 & & & -0.02 \\
\hline \multirow[t]{2}{*}{$50-59$} & Intercept 2000 & -8.85 & 13.14 & 0.02 & 0 & -8.93 & -8.8 \\
\hline & Slope 2000-2015 & -0.02 & & 0 & & & -0.02 \\
\hline \multirow[t]{2}{*}{$60-74$} & Intercept 2000 & -8.3 & 22.1 & 0.02 & 0 & -8.38 & -8.26 \\
\hline & Slope 2000-2015 & -0.03 & & 0 & & & -0.03 \\
\hline \multirow[t]{2}{*}{$75+$} & Intercept 2000 & -7.64 & 42.69 & 0.03 & 0 & -7.73 & -7.59 \\
\hline & Slope 2000-2015 & -0.04 & & 0 & & & -0.03 \\
\hline \multicolumn{8}{|c|}{ Females } \\
\hline \multirow[t]{2}{*}{$0-9$} & Intercept 2000 & -10.888 & 1.508 & 0.039 & 0 & -10.965 & -10.811 \\
\hline & Slope 2000-2015 & -0.038 & & 0.004 & & -0.047 & -0.03 \\
\hline \multirow[t]{2}{*}{ Oct-14 } & Intercept 2000 & -11.129 & 1.301 & 0.068 & 0 & -11.263 & -10.995 \\
\hline & Slope 2000-2015 & -0.072 & & 0.008 & & -0.088 & -0.056 \\
\hline \multirow[t]{2}{*}{$15-19$} & Intercept 2000 & -10.932 & 1.536 & 0.059 & 0 & -11.048 & -10.816 \\
\hline & Slope 2000-2015 & -0.041 & & 0.007 & & -0.054 & -0.028 \\
\hline \multirow[t]{2}{*}{$20-24$} & Intercept 2000 & -11.161 & 1.436 & 0.066 & 0 & -11.29 & -11.033 \\
\hline & Slope 2000-2015 & -0.024 & & 0.007 & & -0.037 & -0.01 \\
\hline $25-29$ & Intercept 2000 & -11.286 & 1.237 & 0.07 & 0 & -11.424 & -11.148 \\
\hline & Slope 2000-2015 & -0.012 & & 0.007 & & -0.027 & 0.002 \\
\hline $30-39$ & Intercept 2000 & -11.037 & 1.623 & 0.049 & 0 & -11.134 & -10.941 \\
\hline & Slope 2000-2015 & -0.027 & & 0.005 & & -0.037 & -0.016 \\
\hline $40-49$ & Intercept 2000 & -10.75 & 2.115 & 0.05 & 0 & -10.847 & -10.652 \\
\hline & Slope 2000-2015 & -0.021 & & 0.005 & & -0.031 & -0.011 \\
\hline $50-59$ & Intercept 2000 & -10.139 & 3.981 & 0.048 & 0 & -10.232 & -10.046 \\
\hline & Slope 2000-2015 & -0.043 & & 0.005 & & -0.053 & -0.033 \\
\hline $60-74$ & Intercept 2000 & -9.441 & 7.145 & 0.036 & 0 & -9.511 & -9.371 \\
\hline & Slope 2000-2015 & -0.037 & & 0.004 & & -0.045 & -0.03 \\
\hline $75+$ & Intercept 2000 & -8.603 & 17.029 & 0.039 & 0 & -8.679 & -8.527 \\
\hline & Slope 2000-2015 & -0.045 & & 0.004 & & -0.053 & -0.037 \\
\hline
\end{tabular}




\section{Juniper Online Journal of Public Health}

Regional Analysis of Pedestrian Fatalities in Older Adults

Next, we analyzed regional fatalities trends in pedestrian aged 60 and over. To explore the performance of each state, we checked if there was a significant increase or decrease in their fatality rates during the period of study (Tables 4-6) and obtained per each state their overall annualized pedestrian death rate (Methods). These metrics allowed us to classify the states in the groups showed in Figure 2. On the upper quadrants of the figure are the states that had an overall annualized pedestrian rate above the national rate obtained in males of 22.95 deaths per 100,000 person/years; on the left are those that presented a significant decrease of their fatality rates across the period of study (negative slope), and on the right, are those that had a significant increase of their fatality rates (positive slope) (Figure 2).

Table 4: Distribution of road traffic fatalities by road user group, Mexico 2000-2015.

\begin{tabular}{|c|c|c|c|c|c|c|}
\hline \multirow{3}{*}{ Year } & \multicolumn{6}{|c|}{ Road Traffic Fatalities } \\
\hline & \multicolumn{3}{|c|}{ Vulnerable } & \multirow[b]{2}{*}{ Protected Users } & \multirow[b]{2}{*}{ Not Specified } & \multirow[b]{2}{*}{ Total } \\
\hline & Pedestrians & Cyclists & Motorcyclists & & & \\
\hline 2000 & 5,509 & 107 & 158 & 8,233 & 20 & 14,027 \\
\hline 2001 & 5,480 & 95 & 198 & 8,222 & 17 & 14,012 \\
\hline 2002 & 5,328 & 116 & 203 & 8,957 & 21 & 14,625 \\
\hline 2003 & 5,348 & 117 & 260 & 9,162 & 24 & 14,911 \\
\hline 2004 & 5,076 & 195 & 288 & 9,431 & 34 & 15,024 \\
\hline 2005 & 5,159 & 186 & 356 & 10,249 & 22 & 15,972 \\
\hline 2006 & 5,220 & 153 & 473 & 10,892 & 29 & 16,767 \\
\hline 2007 & 4,819 & 143 & 588 & 9,779 & 15 & 15,344 \\
\hline 2008 & 4,934 & 174 & 616 & 11,307 & 27 & 17,058 \\
\hline 2009 & 5,147 & 186 & 709 & 11,755 & 19 & 17,816 \\
\hline 2010 & 4,786 & 178 & 704 & 10,861 & 23 & 16,552 \\
\hline 2011 & 4,868 & 152 & 841 & 10,728 & 23 & 16,612 \\
\hline 2012 & 5,177 & 174 & 1,037 & 10,686 & 24 & 17,098 \\
\hline 2013 & 4,816 & 164 & 1,154 & 9,678 & 35 & 15,847 \\
\hline 2014 & 4,821 & 190 & 1,360 & 9,454 & 57 & 15,882 \\
\hline 2015 & 4,573 & 175 & 1,541 & 9,700 & 46 & 16,035 \\
\hline Total & 81,061 & 2,505 & 10,486 & 159,094 & 436 & 253,582 \\
\hline
\end{tabular}

Table 5: Estimates of the parameters obtained by fitting the data of pedestrian casualties among pedestrians' males aged 60 years and over across the different states with a logistic regression model.

\begin{tabular}{|c|c|c|c|c|c|c|c|c|c|c|c|}
\hline \multicolumn{3}{|c|}{ RTI rates } & \multicolumn{9}{|c|}{ Parameters } \\
\hline \multirow{2}{*}{ State } & \multirow{2}{*}{2015} & \multirow{2}{*}{$\begin{array}{c}\text { Overall } \\
\text { annualized } \\
\text { rate }\end{array}$} & \multirow{2}{*}{ B } & \multirow{2}{*}{$\begin{array}{l}\text { Rate } \\
2000\end{array}$} & \multirow{2}{*}{ Sig. } & \multicolumn{2}{|c|}{ CI } & \multirow{2}{*}{ B } & \multirow{2}{*}{ Sig. } & \multicolumn{2}{|c|}{ IC } \\
\hline & & & & & & Inf & Sup & & & Inf & Sup \\
\hline National & 16.58 & 22.96 & -8.09 & 27.28 & $<0.001$ & -8.12 & -8.05 & -0.033 & 0 & -0.037 & -0.03 \\
\hline Aguascalientes & 21.8 & 32.18 & -7.67 & 37.6 & $<0.001$ & -7.96 & -7.38 & -0.42 & 0.007 & -0.072 & -0.012 \\
\hline Baja California & 25.57 & 11.21 & -9.58 & 6.11 & $<0.001$ & -9.97 & -9.19 & 0.049 & 0.005 & 0.014 & 0.083 \\
\hline $\begin{array}{l}\text { Baja California } \\
\text { Sur }\end{array}$ & 17.44 & 19.4 & -8.11 & 31.66 & $<0.001$ & -8.65 & -7.58 & -0.047 & 0.091 & -0.102 & 0.008 \\
\hline Campeche & 4.95 & 14.4 & -8.42 & 18.68 & $<0.001$ & -8.88 & -7.97 & -0.049 & 0.048 & -0.099 & 0 \\
\hline Coahuila & 16.12 & 19.56 & -8.12 & 32.17 & $<0.001$ & -8.34 & -7.89 & -0.048 & 0 & -0.072 & -0.024 \\
\hline Colima & 31.87 & 25.73 & -8.35 & 14.22 & $<0.001$ & -8.78 & -7.93 & 0.01 & 0.643 & -0.031 & 0.05 \\
\hline Chiapas & 21.93 & 7.8 & -11.67 & 2.95 & $<0.001$ & -12.22 & -11.12 & 0.229 & 0 & 0.181 & 0.277 \\
\hline Chihuahua & 15.7 & 20.79 & -8 & 38.27 & $<0.001$ & -8.19 & -7.81 & -0.056 & 0 & -0.076 & -0.035 \\
\hline Mexico City & 18.41 & 35.59 & -7.4 & 50.68 & $<0.001$ & -7.48 & -7.32 & -0.064 & 0 & -0.073 & -0.055 \\
\hline Durango & 27.3 & 23.86 & -8.49 & 15.84 & $<0.001$ & -8.76 & -8.21 & 0.016 & 0.24 & -0.011 & 0.042 \\
\hline
\end{tabular}




\section{Juniper Online Journal of Public Health}

\begin{tabular}{|c|c|c|c|c|c|c|c|c|c|c|c|}
\hline Guanajuato & 19.82 & 38.45 & -7.48 & 52.73 & $<0.001$ & -7.59 & -7.37 & -0.045 & 0 & -0.057 & -0.033 \\
\hline Guerrero & 5.38 & 8.04 & -9.15 & 12.08 & $<0.001$ & -9.43 & -8.86 & -0.033 & 0.034 & -0.064 & -0.002 \\
\hline Hidalgo & 13.7 & 18.33 & -8.31 & 20.77 & $<0.001$ & -8.53 & -8.09 & -0.034 & 0.005 & -0.057 & -0.01 \\
\hline Jalisco & 24.76 & 37.96 & -7.43 & 60.72 & $<0.001$ & -7.53 & -7.34 & -0.052 & 0 & -0.062 & -0.042 \\
\hline México & 14.75 & 23.93 & -7.99 & 28.89 & $<0.001$ & -8.09 & -7.9 & -0.038 & 0 & -0.048 & -0.028 \\
\hline Michoacán & 7.82 & 16 & -8.14 & 25.03 & $<0.001$ & -8.3 & -7.97 & -0.075 & 0 & -0.094 & -0.056 \\
\hline Morelos & 8.21 & 17.96 & -8 & 23.36 & $<0.001$ & -8.26 & -7.75 & -0.075 & 0 & -0.103 & -0.046 \\
\hline Nayarit & 17.54 & 23.86 & -8.07 & 11.7 & $<0.001$ & -8.36 & -7.78 & -0.031 & 0.043 & -0.061 & -0.001 \\
\hline Nuevo León & 7.19 & 15.4 & -8.73 & 0.78 & $<0.001$ & -8.95 & -8.52 & -0.005 & 0.652 & -0.025 & 0.016 \\
\hline Oaxaca & 11.77 & 11.78 & -9.29 & 5.96 & $<0.001$ & -9.53 & -9.05 & 0.027 & 0.023 & 0.004 & 0.05 \\
\hline Puebla & 10.5 & 22.75 & -7.69 & 30.65 & $<0.001$ & -7.82 & -7.57 & -0.086 & 0 & -0.101 & -0.071 \\
\hline Querétaro & 27.23 & 36.63 & -7.61 & 37.62 & $<0.001$ & -7.84 & -7.39 & -0.033 & 0.004 & -0.056 & -0.011 \\
\hline Quintana Roo & 13.21 & 10.21 & -8.86 & 22.44 & $<0.001$ & -9.48 & -8.23 & -0.035 & 0.262 & -0.097 & 0.026 \\
\hline San Luis Potosí & 25.25 & 27.42 & -8.24 & 12.36 & $<0.001$ & -8.43 & -8.05 & 0.004 & 0.646 & -0.014 & 0.023 \\
\hline Sinaloa & 26.79 & 27.17 & -8.37 & 21.33 & $<0.001$ & -8.57 & -8.17 & 0.017 & 0.072 & -0.002 & 0.036 \\
\hline Sonora & 10.81 & 21.68 & -8.44 & 19.1 & $<0.001$ & -8.67 & -8.2 & 0 & 0.999 & -0.022 & 0.022 \\
\hline Tabasco & 32.94 & 34.47 & -8.09 & 21.61 & $<0.001$ & -8.31 & -7.88 & 0.013 & 0.209 & -0.007 & 0.034 \\
\hline Tamaulipas & 22.59 & 28.59 & -7.96 & 36.68 & $<0.001$ & -8.14 & -7.79 & -0.022 & 0.014 & -0.04 & -0.004 \\
\hline Tlaxcala & 20.77 & 25.4 & -8.53 & 21.04 & $<0.001$ & -8.87 & -8.19 & 0.028 & 0.092 & -0.004 & 0.06 \\
\hline Veracruz & 9 & 12.15 & -8.81 & 12.47 & $<0.001$ & -8.97 & -8.66 & -0.023 & 0.005 & -0.039 & -0.007 \\
\hline Yucatán & 19.25 & 19.54 & -8.28 & 18.92 & $<0.001$ & -8.53 & -8.04 & -0.029 & 0.023 & -0.055 & -0.004 \\
\hline Zacatecas & 23.73 & 22.24 & -8.17 & 39.76 & $<0.001$ & -8.42 & -7.92 & -0.029 & 0.033 & -0.055 & -0.002 \\
\hline
\end{tabular}

Table 6: Estimates of the parameters obtained by fitting the data of pedestrian casualties among pedestrians' females aged 60 years and over across the different states with a logistic regression model.

\begin{tabular}{|c|c|c|c|c|c|c|c|c|c|c|c|}
\hline \multicolumn{3}{|c|}{ RTI rates } & \multicolumn{9}{|c|}{ Parameters } \\
\hline \multirow{2}{*}{ State } & \multirow{2}{*}{2015} & \multirow{2}{*}{$\begin{array}{c}\text { Overall } \\
\text { annualized } \\
\text { rate }\end{array}$} & \multirow{2}{*}{ B } & \multirow{2}{*}{$\begin{array}{l}\text { Rate } \\
2000\end{array}$} & \multirow{2}{*}{ Sig. } & \multicolumn{2}{|c|}{ CI } & \multirow{2}{*}{ B } & \multirow{2}{*}{ Sig. } & \multicolumn{2}{|c|}{ IC } \\
\hline & & & & & & Inf & Sup & & & Inf & Sup \\
\hline National & 5.37 & 7.6 & -9.13 & 9.84 & 0 & -9.18 & -9.08 & -0.04 & 0 & -0.045 & -0.035 \\
\hline Aguascalientes & 5.03 & 13.5 & -8.63 & 20.85 & 0 & -9.06 & -8.21 & -0.031 & 0.162 & -0.074 & 0.012 \\
\hline Baja California & 10.69 & 3.3 & -11.72 & 1.38 & 0 & -12.68 & -10.76 & 0.139 & 0 & 0.065 & 0.212 \\
\hline $\begin{array}{c}\text { Baja California } \\
\text { Sur }\end{array}$ & & 5.1 & -9.71 & & 0 & -10.94 & -8.48 & 0.039 & 0.526 & -0.083 & 0.161 \\
\hline Campeche & & 2.8 & -9.18 & 8.67 & 0 & -10.06 & -8.3 & -0.084 & 0.196 & -0.21 & 0.043 \\
\hline Coahuila & 1.96 & 5.5 & -9.44 & 9.41 & 0 & -9.85 & -9.04 & -0.041 & 0.057 & -0.083 & 0.001 \\
\hline Colima & 10.65 & 6.6 & -9.62 & & 0 & -10.53 & -8.7 & 0.01 & 0.82 & -0.074 & 0.093 \\
\hline Chiapas & 5.68 & 1.9 & -12.13 & 2.43 & 0 & -13.27 & -10.99 & 0.159 & 0.001 & 0.061 & 0.256 \\
\hline Chihuahua & 5.27 & 7 & -9.05 & 11.17 & 0 & -9.36 & -8.75 & -0.058 & 0.001 & -0.092 & -0.025 \\
\hline Mexico City & 9.73 & 14.9 & -8.42 & 19.87 & 0 & -8.53 & -8.32 & -0.045 & 0 & -0.057 & -0.034 \\
\hline Durango & 7.56 & 6.6 & -9.94 & 5.29 & 0 & -10.48 & -9.4 & 0.033 & 0.2 & -0.017 & 0.082 \\
\hline Guanajuato & 5.08 & 10.5 & -8.69 & 13.27 & 0 & -8.89 & -8.5 & -0.054 & 0 & -0.076 & -0.033 \\
\hline Guerrero & 2.58 & 4 & -9.71 & 7.23 & 0 & -10.09 & -9.33 & -0.04 & 0.048 & -0.081 & 0 \\
\hline Hidalgo & 1.27 & 4.8 & -9.86 & 2.92 & 0 & -10.29 & -9.43 & -0.01 & 0.653 & -0.053 & 0.033 \\
\hline Jalisco & 7.67 & 11.9 & -8.52 & 19.26 & 0 & -8.67 & -8.37 & -0.06 & 0 & -0.077 & -0.043 \\
\hline México & 5.29 & 8.5 & -9.02 & 9.45 & 0 & -9.17 & -8.88 & -0.038 & 0 & -0.053 & -0.023 \\
\hline Michoacán & 1.51 & 6.2 & -8.86 & 10.61 & 0 & -9.1 & -8.62 & -0.106 & 0 & -0.136 & -0.076 \\
\hline Morelos & 1.75 & 7.1 & -9.16 & 8.49 & 0 & -9.55 & -8.76 & -0.046 & 0.031 & -0.088 & -0.004 \\
\hline Nayarit & 3.02 & 7.3 & -8.97 & & 0 & -9.52 & -8.41 & -0.048 & 0.097 & -0.104 & 0.009 \\
\hline
\end{tabular}


Juniper Online Journal of Public Health

\begin{tabular}{|c|c|c|c|c|c|c|c|c|c|c|c|}
\hline Nuevo León & 1.11 & 3.5 & -9.52 & & 0 & -9.93 & -9.12 & -0.075 & 0.001 & -0.118 & -0.033 \\
\hline Oaxaca & 3.69 & 3.9 & -10.19 & 2.77 & 0 & -10.56 & -9.81 & 0.005 & 0.81 & -0.033 & 0.042 \\
\hline Puebla & 5.74 & 9.3 & -8.67 & 16.92 & 0 & -8.86 & -8.48 & -0.073 & 0 & -0.095 & -0.052 \\
\hline Querétaro & 10.97 & 11.4 & -8.81 & 17.15 & 0 & -9.18 & -8.44 & -0.03 & 0.115 & -0.068 & 0.007 \\
\hline Quintana Roo & 8.72 & 2.4 & -9.64 & & 0 & -11.02 & -8.26 & 0.021 & 0.737 & -0.103 & 0.145 \\
\hline San Luis Potosín & 7.8 & 7 & -9.67 & 2.84 & 0 & -10.07 & -9.27 & 0.016 & 0.401 & -0.021 & 0.054 \\
\hline Sinaloa & 7.82 & 6.2 & -10.14 & 6.32 & 0 & -10.58 & -9.7 & 0.047 & 0.019 & 0.008 & 0.087 \\
\hline Sonora & 5.3 & 5.7 & -9.61 & 6.14 & 0 & -10.03 & -9.18 & -0.019 & 0.385 & -0.061 & 0.023 \\
\hline Tabasco & 10.34 & 6.1 & -10.01 & 4.9 & 0 & -10.54 & -9.47 & 0.032 & 0.204 & -0.017 & 0.081 \\
\hline Tamaulipas & 6.85 & 7.2 & -9.11 & 12 & 0 & -9.42 & -8.8 & -0.049 & 0.004 & -0.081 & -0.016 \\
\hline Tlaxcala & 1.62 & 6.1 & -9.19 & 12.41 & 0 & -9.74 & -8.63 & -0.062 & 0.048 & -0.124 & -0.001 \\
\hline Veracruz & 1.6 & 3.8 & -9.77 & 4.93 & 0 & -10.02 & -9.52 & -0.048 & 0.001 & -0.075 & -0.021 \\
\hline Yucatán & 4.12 & 4.8 & -9.53 & 2.76 & 0 & -10 & -9.06 & -0.049 & 0.056 & -0.099 & 0.001 \\
\hline Zacatecas & 6.82 & 6.4 & -9.06 & 7.92 & 0 & -9.49 & -8.63 & -0.065 & 0.011 & -0.116 & -0.015 \\
\hline
\end{tabular}

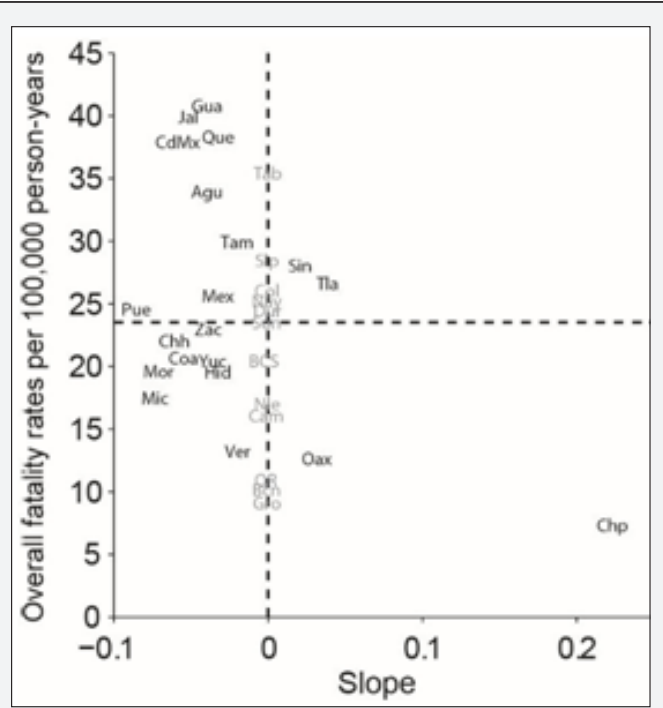

Figure 2 : Correlation between the annualized fatal rates per each state and the slope's value obtained in the group of pedestrian males aged 60 and over, Mexico, 2000-2014, Agu, Aguascalientes; Bcn, Baja California Norte; BCS, Baja California Sur; Cam, Campeche; Coa, Coahuila; Col, Colima; Chp, Chiapas; Chh, Chihuahua; CdMx, Ciudad de México; Dur, Durango; Gua, Guanajuato; Gro, Guerrero; Hid, Hidalgo; Jal, Jalisco; Mex, Estado de México; Mic, Michoacán; Mor, Morelos; Nay, Nayarit; Nle, Nuevo León; Oax, Oaxaca; Pue, Puebla; Que, Querétaro; Roo, Quintana Roo; Slp, San Luis Potosí; Son, Sonora; Tab, Tabasco; Tam, Tamaulipas; Tla, Tlaxcala; Ver, Veracruz; Yuc, Yucatán; Zac, Zacatecas.

For the group of males aged 60 years and older, overall annualized pedestrian rates of Colima, Durango, San Luis Potosí, Sinaloa, Tabasco and Tlaxcala, were among the highest during the period, and their RTI rates remained constant during all period (Figure 2) upper quadrant, gray letters. Some states also presented an overall annualized rate above the overall annualized rate of 22.9 deaths per 100,000 person/years, such as Aguascalientes, Mexico City, Guadalajara, Jalisco, State of Mexico, Nayarit, Querétaro and Tamaulipas, however, their mortality rates had a significant tendency towards decreasing.
On the other hand, there are states with an overall annualized fatality rate below the national rate for males, on the right lower quadrant (Figure 2) are shown those that had a significative tendency towards increasing their fatality rates: Oaxaca, Baja California and Chiapas; on the axis of ordinates are shown those in which RTI rates remained constant during the period (Figure 2 ); lower quadrant, gray letters), and finally on the left lower quadrant are shown those states $34.4 \%(n=11)$ that have a significative tendency towards decreasing their RTI rates.

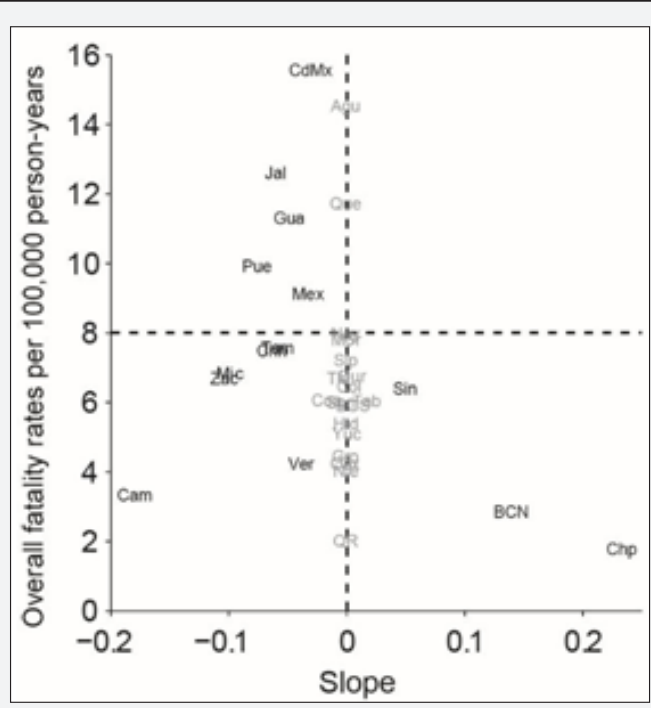

Figure 3 : Correlation between the annualized fatal rates (period 2000-2014) per each state and the slope's value obtained in the group of pedestrian females aged 60 and over.

Also, we carried out this analysis in the females' group (Figure 3). When comparing the scale's figures obtained in both groups, is evident their contrast, in the sense that the overall fatality rates in males are by far higher than the obtained among females In the group of females, in 15 (46.9\%) states their RTI rates remained constant over the period of study (Figure 3) gray letters, 2 of those states: Aguascalientes and Queretaro had an overall annualized rate above the calculated at national level in 
females of 7.6 deaths per 100,000 person/years. Note that only 3 states showed a tendency toward the increase of their mortality rates: Sinaloa, Baja California Norte, and Chiapas (Figure 3) right lower quadrant, however, their overall annualized pedestrian rate is below the national one.

\section{Discussion}

In this work we analyzed the demographic, spatial and temporal characteristics of fatal traffic accidents involving pedestrians. In particular, we focus on the study of elderly pedestrians, who according to the literature constitute a group vulnerable to traffic accidents due to the decrease of their cognitive and perceptual abilities. Between the years 2000 and 2015, pedestrians contributed with almost a third of the total deaths due to traffic accidents in Mexico, which ranked second nationally among road users with the highest number of deaths (after the occupants of motor vehicles). However, in Mexico City, pedestrians were the main fatal victims of traffic accidents between 2000 and 2015. It should be noted that the percentage that represented pedestrian deaths in Mexico (32\%) is higher than the percentage observed globally and in the Americas region (22\%) [4]. On the contrary, the distribution of deaths in these users by age group coincides with that reported by other countries (OECD \& ITF, 2016). In both sexes, it was observed that as the age increases also the risk of suffering a fatal accident as a pedestrian, in such a way that the highest rates were observed in the age groups: 60-75 years and 75 and over. Despite the foregoing, to date there have been few studies in Mexico on safety and mobility López, 2014, ONU-Habitat 2015 in this group of users of public roads, so it seemed important to analyze the distribution regional in this age group with the aim of identifying in which states have to focus preventive actions. The indicators used allowed us to determine in which states there is a significant reduction in their mortality rates throughout the period and which of them are above the annualized incidence rate at the national level. Based on the indicators used, we observe a great heterogeneity among the states. In the group of men aged 60 years and over, in 19 (59.3\%) states there is a significant trend towards decreasing their mortality rates, in 10 states $(31.2 \%)$, mortality rates remained more or less constant and in 3 states there is a tendency towards the rise.

The above can be a reflection of the different level of application of measures in road safety, at the state and municipal level. While in some Mexican cities their legislation on permitted blood alcohol levels has been strengthened or has worked closely with the highway police to hold workshops on the good practices for the prevention of risk factors, in other States such as Colima, Durango, San Luis Potosi, Sinaloa, Tlaxcala and Tabasco, whose annualized rate was one of the highest, and where their mortality rates have remained fluctuating for In the following years, only in Tabasco, its road safety legislation takes into consideration the 6 risk factors considered by WHO as minimums, while in Durango, for example, its road safety legislation does not mention the same. In addition, at the municipal level, legislation on road safety may vary [16]. Until December 2015, some adjustments were put into effect to include the priority risk factors in one of the municipalities of Tlaxcala, while in the rest of the municipalities the application level has not yet been revised.

Likewise, in addition to the risk factors considered minimal by the WHO, other key measures aimed at reducing pedestrian accidents still need to be adopted and effectively applied. Recently a group of experts analyzed the scientific evidence and recommended a series of measures that contribute to reduce the frequency and severity of traffic accidents, among which are; speed control, effective regulation of safety devices in automobiles, design and improvement of infrastructure, surveillance in compliance with laws, emergency care after a traffic accident and leadership. In relation to speed, important advances have been made in some cities by monitoring over speed, among which are: Mexico City, Puebla, Jalisco, Veracruz, Pachuca De Soto, Aguascalientes and the municipality of García in Nuevo León. Coincidentally, in most of these states a sustained reduction in mortality rates has been reached in pedestrians of 60 and over, except in the State of Nuevo León, where mortality rates in Men pedestrians over 60 have been fluctuating.

With respect to automobile safety standards, the New Vehicle Evaluation Program for Latin America and the Caribbean states that most vehicles sold in Mexico do not comply with international minimum safety standards, while the Mexican Standard regulates essential safety devices for new vehicles, is late in comparison to other countries since it will come into force until 2020 and does not incorporate all the elements considered essential as electronic stability control. Another measure that could contribute to the reduction of accidents in this group is the design and improvement of road infrastructure considering the needs of vulnerable users. However, in Mexico the infrastructure for motorized transport has been prioritized, and until recently, some Mexican cities have included in their legislation sustainable mobility as a right and have established that the pedestrian is the user with the highest priority in public space.

To cite some examples, the Mobility Law of the Federal District was approved only in July of 2014 and the Mobility Law of the State of Mexico was published in August 2015. This suggests that there is still much to be done in terms of mobility and safety for vulnerable road users. About the limitations of our study it is important to mention that, to estimate the risk of mortality in pedestrians, the population was used as the denominator since there is little information in Mexico about mobility by type of user of the public road. The data generated by the Origin-Destination Surveys are not available for all the years analyzed and are mainly concentrated for trips made by residents of the metropolitan area of Mexico City. Another aspect that is important to highlight is the quality of mortality records in Mexico, since in most fatal accidents involving pedestrians 
there is no information on the mechanism of the injury. This contrasts with the records of fatal accidents in other countries such as England or the United States, where it is known that most pedestrian deaths were derived from a collision with a motor vehicle.

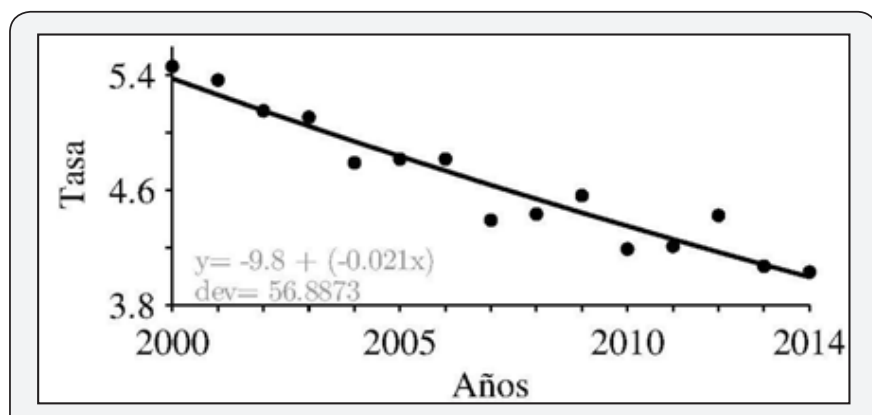

Figure 4 : Temporal evolution of RTI rates at national level.

Another limitation of the study relates to under reporting and misclassification of road deaths and collision mechanisms among pedestrians, due to inappropriate and nonspecific ICD10 coding of RTI deaths at state level. The underestimation of RTI's could represent a substantial underestimation in the mortality burden currently estimated for the country. Recent studies have documented that mortality from RTI's in Mexico, during the period between 1999 and 2013, is underestimated by $18.85 \%$, with important variations at state level [17-20]. The actual burden of road traffic mortality in Mexico, as reported by nationally collected data, is underestimated due to inappropriate and nonspecific ICD -10 coding of RTI deaths (Figure 4) [21,22].

\section{Conclusion}

With continued urbanization and motorization, VRU injuries and deaths represent a serious threat to public health and development in Mexico, and jeopardize the pursuit of equity in health, because they disproportionately affect the urban poor. Previous studies have evidenced, that between 1990 and 2010, there has been an increase in healthy years of life lost due to motor vehicle crashes and a decrease in life expectancy among Mexican men. The proportion of RTI deaths among pedestrians is much higher in Mexico than what is observed in the world, or in the region of the Americas. Although mortality rates in all sex-age groups have decreased during the period, there are still high death rates in pedestrian males aged 60 years and over. This suggests that specific measures are required to ensure safe urban mobility for this group, particularly in the states with an increasing trend in mortality.

It has been recognized that a prerequisite for ameliorating RTI's and preventing road traffic deaths is good quality data that is widely disseminated. Therefore, Mexico must harness efforts to improve ICD coding. Also evident, is the need for Mexico to construct safer roads, create more rigorous rules regarding road safety, and enforce existing laws in order to reduce mortality from RTI's. Road traffic safety is the quintessential issue that demands a whole of government/whole of society approach. A medical perspective is not wholly appropriate for guiding road safety interventions and thus, a larger, multidisciplinary approach to promote road safety is important. Mexico must continue to emphasize that motor vehicle trauma, especially to pedestrians, is an ongoing public health epidemic, and must ensure strategies to reduce pedestrian risk in urban settings that are informed by science and implemented with comprehensive stakeholder involvement.

Although the proportion of fatal accidents in pedestrians in Mexico is high compared to the proportion represented by fatal accidents in pedestrians at the global level, or in the region of the Americas, it was found that there is a significant tendency towards the decrease in their rates of mortality, for almost all age-sex groups analyzed. Among pedestrians, there are some demographic groups that are at greater risk of suffering a fatal accident. In men the risk of suffering a fatal accident is greater and this risk increases according to age, in such a way that the highest mortality rates were observed in men over 60 years of age. The high mortality rates among pedestrians over 60 needs to be addressed by the authorities involved. In particular, it is necessary to carry out studies at the micro local or intersection level in the States whose tendency is to increase and strengthen the implementation of public policies on road safety. Walking as a mode of transportation has received little attention during the planning and development of policies in Mexico. Therefore, it is necessary that a mobility policy that promotes non-motorized modes of transport with a pedestrian-based approach be promoted at the national and sub-national levels.

\section{References}

1. Mathers CD, Loncar D (2006) Projections of global mortality and burden of disease from 2002 to 2030. PLoS Medicine 3(11): e442.

2. Sharma B (2008) Road traffic injuries: a major global public health crisis. Public Health 122(12): 1399-1406.

3. World Health Organization (2009) Global status report on road safety. Time for action.

4. WHO (2015) Global status report on road safety. Summary, WHO, Geneva, Switzerland.

5. Constant A, Lagarde E (2010) Protecting Vulnerable Road Users from Injury. Plos Medicine 7(3): e1000228.

6. Organization PAH (2016) Road Safety in the Americas. Washington, USA, p.30.

7. Cervantes Trejo A, Leenen I, Fabila Carrasco JS, Rojas Vargas R (2016) Trends in traffic fatalities in Mexico: examining progress on the decade of action for road safety 2011-2020. International Journal of Public Health 61(8): 903-913.

8. Koehlmoos TP (2013) Why Aren't We Listening Yet? A Decade of Road Safety Begins Quietly. Plos Medicine 10(6): e1001464.

9. Wegman F (2017) The future of road safety: A worldwide perspective. IATSS Research 40(2): 66-71.

10. INEGI (2014) General deaths statistics Methodological synthesis.

11. WHO (2011) International statistical classification of diseases and related health problems ( $10^{\text {th }}$ revision). Geneva, Switzerland. 
12. CONAPO (2012) Proyecciones de la Población 2010-2050. CONAPO.

13. Dobson AJ, Barnett A (2008) An Introduction to Generalized Linear Models. ( $3^{\text {rd }}$ edn.); Chapman and Hall/CRC, Boca Raton, Florida, USA, pp.320.

14. The R Development Core Team (2013) R: A language and environment for statistical computing. R Foundation for Statistical Computing, Vienna, Australia.

15. Mendenhall W, Sincich TL (1995) Statistics for Engineering and the Sciences. ( $4^{\text {th }}$ edn.); Englewood Cliffs, Prentice Hall, NJ, USA.

16. Pérez Núñez R, Mojarro Íñiguez MG, Mendoza García M, Rosas Osuna SR, Híjar M (2016) Underestimation of mortality caused by transit in Mexico: subnational analysis 58(4): 412-420.

17. Híjar M, Chandran A, Pérez Núñez R, Lunnen JC, Martín Rodríguez Hernández J, et al. (2012) Quantifying the underestimated burden of road traffic mortality in Mexico: a comparison of three approaches. Traffic Injury Prevention 13(1): 5-10.
18. Rodrigues E (2016) Road Safety in the Region of the Americas. Pan American Health Organization, editor, Pan American Health Organization, Washington, USA

19. González Pérez GJ, Vega López MG, Cabrera Pivaral CE (2015) Mortality due to traffic injuries and its impact on life expectancy: a comparison between Mexico and Spain. Gaceta Sanitaria 29(Suppl 1): 70-75.

20. Barbour V, Clark J, Jones S, Peiperl L, Veitch E (2010) Preventing Road Deaths-Time for Data. Plos Medicine 7(3): e1000257.

21. Redelmeier DA, McLellan BA (2013) Modern Medicine Is Neglecting Road Traffic Crashes. Plos Medicine 10(6): e1001463.

22. Bliss T, Breen J (2012) Meeting the management challenges of the Decade of Action for Road Safety. IATSS Research 35(2): 48-55.

\section{Your next submission with Juniper Publishers will reach you the below assets}

- Quality Editorial service

- Swift Peer Review

- Reprints availability

- E-prints Service

- Manuscript Podcast for convenient understanding

- Global attainment for your research

- Manuscript accessibility in different formats

( Pdf, E-pub, Full Text, Audio)

- Unceasing customer service

Track the below URL for one-step submission https://juniperpublishers.com/online-submission.php 